\title{
Neuropsicología de la empatía
}

\section{Neuropsychology of empathy}

\author{
Sara Raquel Romero Godoy
}

Málaga

Máster en Neuropsicología Clínica. ATEN-D

Correspondencia: srromerogodoy@gmail.com

Resumen: La empatía ha sido ampliamente estudiada por numerosas disciplinas a lo largo de la historia, pero a pesar de ello en la actualidad no hay una base clara y unificada de lo que es la empatía y las bases neuronales de la misma. Objetivo: Unificar los diferentes conceptos de empatía y revisar las distintas bases neuronales descubiertas hasta la actualidad con la finalidad de poder recopilar, esclarecer y sintetizar todos los descubrimientos. Con ello se pretende alcanzar una visión clara y concisa de lo que es la empatía y que mecanismos anatomofisiológicos son los encargados de la misma. Desarrollo: La visión actual de la empatía es multidimensional, estando integrada por un componente afectivo, las neuronas espejo principalmente, y por un componente cognitivo, que es la Teoría de la Mente. Conclusión: El componente afectivo está constituido por una cadena de neuronas espejo que es el componente esencial y en el que participa la imitación, la comprensión y el lenguaje. El componente cognitivo está ligado a la corteza prefrontal principalmente y a la encrucijada temporo-parietal (en adelante ETP).

Palabras clave: Empatía, Emociones, Neuropsicología, Función Ejecutiva, Neuronas Espejo.
Abstract: The empathy has been widely studied by many disciplines along the history, but in spite of it is not clear what is empathy and its neuronals. Objective: Unify the different concepts of empathy and review the various neural basis discovered until now in order to be able to gather, clarify and summarize all findings. This is to achieve a clear and concise vision of what is empathy and anatomophysiological mechanisms are responsible for the same. Development: The current view of empathy is multidimensional, being composed of an affective component, mirror neurons mainly, and a cognitive component, which is the Theory of Mind. Conclusion: The affective component is constituted by a chain of mirror neurons is the essential component and in which participates imitation, comprehension and language. The cognitive component is linked primarily to the prefrontal cortex and temporo-parietal juncture (ETP).

Keywords: Empathy, Emotions, Neuropsychology, Executive Functions, Mirror Neurons. 


\section{Introducción}

Para poder definir empatía recurriremos inicialmente al origen de la palabra, que como muchas proceden del griego, empatheia, que significa sentir o sentir dentro [1].

Pero para entender un poco mejor qué entendemos con este término podemos decir a modo general que es una habilidad que de los seres humanos para poder llegar a comprender los sentimientos y emociones de las personas que nos rodean, puede decirse que es como "ponernos en el lugar de otra persona pero sin salir de nuestro propio ser". Conlleva dos procesos consecutivos, impregnarse de los sentimientos y emociones de otras personas y comprenderla como individuo cuya mentalidad es distinta a nosotros [2].

Hemos de tener en cuenta que son muchos los autores que han empleado desde entonces el término de empatía, pero no todos ellos lo han hecho con el mismo enfoque, ni han detallado los mismos procesos básicos, de ahí la gran confusión en cuanto a concreción de definición y en cuanto a procesos cognitivos y estructuras ligadas a los mismos [2].

Este concepto llega al mundo de la psicología a principios del siglo XX de la mano de Lipps (1903) y a partir de entonces se extiende por diversas ramas de la ciencia, psicoterapia y más tarde a la neuropsicología. Dicho autor la concebía como un proceso automático por medio del cual cuando el sujeto percibía una emoción de otra persona a través de sus gestos y, a través de ello, se producía una activación directa de dicha emoción percibida [2]. En 1904 la palabra empathy aparece en el diccionario inglés de Webster y, en alemán Einfühlung es utilizado por Sigmund Freud en sus escritos y, lo considera como la fusión de lo que ve y siente el sujeto lo cual, tiene lugar al proyectar el sujeto sus sentimientos sobre el objeto intuido [3].

A mediados del siglo XX aproximadamente, surgen otras concepciones del término. En 1958 Aring la define como una acción o capacidad para apreciar los sentimientos de otra persona pero sin participar en ellos [4]. Un año más tarde Rogers la define como una habilidad para poder percibir la estructura interna de referencia de otro individuo de forma precisa "como si" fuera esa otra persona pero sin perder esa condición del "como si" [5].

A finales del siglo XX, en 1981 Hoffman tiende a considerar la empatía como respuesta indirecta e involuntaria a la reacción afectiva de otra persona [6]. En 1990 Zahn-Waxler define la empatía teniendo en cuenta componentes afectivos y cognitivos, además considera a la empatía como un componente esencial para comprender al individuo [7]. Gold, en 1995 considera la considera como una percepción del estado interno de referencia del otro [8]. En 1996 salen a la luz dos nuevas conceptualizaciones, por un lado la de Meharian, que considera la empatía emocional como la experiencia de las emociones de otra persona, y a su vez afirma que el tenerla es símbolo de saludabilidad, personalidad ajustada, seguridad y habilidad en las relaciones interpersonales [9]. Cohen en ese mismo año afirma que es una habilidad para comprender y compartir el estado emocional de otro individuo [10]. Davis, en 1996, argumenta que es un tipo de conducta que promueve la comunicación, las relaciones personales así como la resolución de conflictos [11]. En 1997, Brohart considera a la empatía como el llegar a sentir lo mismo que otra persona siente, y por lo tanto ser un constructor esencial en la óptima relación social [12].

A comienzos del siglo XXI surge el modelo de Percepción /Acción de Preston y Waal en 2002 y, la Teoría de la Simulación de Gallese en 2001 concebían que existiera percepción automática de emociones como 
base de la empatía [13,14]. En 2002 Hojat considera esencial tres factores primarios en la empatía: atención, compasión y habilidad para ponerse en el lugar del otro [15,16]. Baron-Cohen en 2003 concibe la empatía como una capacidad tanto para identificar emociones y pensamientos de otra persona como de poder responder con la adecuada emoción sin apenas realizar esfuerzo [17].

En el año 2004 surgen dos concepciones, una de la mano de González de Rivera define a la empatía como una capacidad para comprender y experimentar vicariamente los sentimientos, pensamientos y experiencias de otra persona sin que estos hayan sido comunicados de forma explícita [18]. La otra concepción que surge en este año es el modelo de Decety y Jackson (2004), que incluye tres componentes que interactúan dinámicamente para producir la empatía humana: 1) la emoción compartida entre el observador y el observado, es decir, la respuesta afectiva más automática; 2) la autoconciencia y conciencia del otro, que impide la confusión entre el self y el otro a pesar de cierta identificación temporal; y 3) la flexibilidad cognitiva para adoptar la perspectiva del otro, que requiere de procesos de regulación. De acuerdo con estos autores, ninguno de los tres componentes puede dar cuenta por sí mismo del potencial de la empatía humana, y en el funcionamiento normal están interrelacionados [19].

Blair en 2005 propone tres componentes de la empatía parcialmente disociables e independientes: 1) empatía cognitiva (Teoría de la mente o ToM, en adelante) 2) empatía motriz o neuronas en espejo 3) empatía emocional [20].

Decety y Lamm en el año 2006 proponen las meta-categorías bottom-up (procesos de percepción) y topdown (procesos de regulación y control) para diferenciar de modo más global los distintos procesos internos del modelo mencionado [21].

En el presente artículo realizamos una aproximación de los mecanismos neuropsicológicos y neurofisiológicos del proceso de la empatía desde una dimensión cognitiva y afectiva.

\section{Objetivo}

El objetivo general de este estudio es realizar una revisión de las bases neuropsicológicas y neuroanatómicas de la empatía con el fin de recopilar datos para esclarecer su constructo y su localización cerebral.

La búsqueda bibliográfica se ha realizado mediante una serie de bases de datos: Pubmed, y Medline. Así como en diversas revistas de gran impacto como son neuroimagen, Nature, Neurscience y Brain entre otras. Los términos en inglés introducidos para la realización de la búsqueda fueron: "empathy", "neuropsychology of empathy" y "neuroanatomy of empathy".

\section{Desarrollo}

Inicialmente se consideró que ambos tipos de empatía estaban ligados a las mismas estructuras anatómicas pero, existen nuevas evidencias de que están en circuitos neuronales diferenciados. Así pues el área que corresponde a la región opercular del giro frontal inferior (área 44 de Broadmann) mostró que era importante para la empatía emocional y, por otra parte las regiones que fueron de especial relevancia para la empatía cognitiva fueron las áreas anteriores del giro frontal superior y medio, y del giro orbital, el recto y la zona más anterior del giro superior frontal (áreas 10, 11 de Broadmann, respectivamente) [22, 23]. 
Los estudios más actuales de la empatía han promovido la fusión de todos los procesos en un constructo amplio. Todos estos modelos tratan de combinar la experiencia afectiva automática con el estado emocional percibido con la comprensión de los estados emocionales de los demás a través de procesos cognitivos controlados, para dar lugar a esquemas comprensivos de los estados emocionales observados [23].

La parte cognitiva está íntimamente relacionado con la ToM, que es la capacidad para poder abstraer los procesos mentales de otras personas. Por otro lado, el componente emocional de la empatía es el resultado de tratar de comprender a otra persona hasta el punto de ponerse en el lugar del otro, dando como resultado acercarse al estado emocional del otro y reaccionar. Esta parte emocional la podemos relacionar con las neuronas espejo. Además, éste último tipo de empatía lo podemos dividir en dos subgrupos: uno más relacionado con la expresión emocional de ira y rabia, y otro más ligado a expresiones de miedo o tristeza $[24,25]$.

Las diversas investigaciones que se han realizado en el campo de la empatía se han llevado a cabo teniendo en cuenta la presentación de estímulos: emocionales, expresión de asco, somato sensoriales y de dolor [26].

Con respecto a los estímulos emocionales podemos destacar el trabajo de Geday et al [27] en el que llega a la conclusión que el área fusiforme posterior identifica numerosas señales emocionales importantes para la percepción social. Y que además éste área es una de las que envía información a la corteza prefrontal medial inferior derecha, constituyendo una red neuronal que es de especial relevancia para las interacciones sociales y la empatía.

Otra estructura relevante en el procesamiento emocional es la ínsula, que se encarga del entendimiento de los sentimientos de los demás mediante un mecanismo de representación emocional que permite la empatía y regula el contenido emocional [28].

Los estímulos somato sensoriales también se ven implicados dentro de los mecanismos de la empatía. Ello pudo verse en un estudio en el que se valoró como se activaba la corteza somato sensorial secundaria cuando algunos individuos veían películas en las que a algún personaje se le tocaba la pierna [29].

Teniendo en cuenta la expresión de asco, se ha observado que hay diversas estructuras implicadas: la ínsula anterior y el opérculo frontal adyacente, conjunto de estructuras que se denominan (en adelante IFO) y que están implicadas en la empatías de las emociones positivas y negativas [30, 31, 32]. Diversos estudios con pacientes con lesión amigdalina han demostrado que la amígdala también contribuye a la percepción de estímulos negativo (relación de estímulos sensoriales de afecto y la generalización de diferentes emociones) como el miedo, la ira o el asco, así como también se la ha relacionado con la función de identificación de las expresiones faciales emocionales y mediación del nivel de arousal vegetativo [33].

\section{Componente emocional de la empatía}

El lenguaje es el medio mediante el que nos comunicamos con el resto de seres humanos y en algunos casos si es primitivo y rudimentario con los animales de otras especies. Cuando el ser humano llega a la edad adulta los gestos que emite durante su discurso podrían clasificarse en dos tipos: rítmicos e icónicos. Los primeros son aquellos que reflejan el contenido del discurso al que acompañan, en cambio los 
segundos no reflejan de forma específica o visual aquello que se dice, sino que son movimientos de la mano que aparecen casi para marcar la musicalidad del habla. Martha Alibali y sus colaboradores estudiaron que las personas que hablaban por teléfono tendían a reducir la cantidad de gestos icónico, mientras que la frecuencia de los gestos rítmicos no se vio alterada. Puede deducirse de esto, que los gestos rítmicos tienen más utilidad para el hablante, mientras que los gestos icónicos son más importantes para el receptor/oyente [34]. Tras la verificación de este suceso, Istvan Molnar-Szakacs planteó la hipótesis de que las neuronas espejos facilitan la comunicación, y en consecuencia, la activación de las mismas sería mayor cuanto mayor sea la observación de gestos icónicos, los cuales favorecen la comunicación y comprensión. Por otra parte, como cabe esperar, la activación de estas neuronas sería nula cuando la persona observaba gestos rítmicos [35].

En estudios posteriores se llegó a la conclusión de que estos gestos icónicos que estaban ligados a las neuronas espejo eran utilizados en edades tempranas, con lo que se generó la hipótesis de que las neuronas espejo son críticas en el desarrollo y evolución del lenguaje [36]. Esto se ve íntimamente ligado a la concepción que se tiene de que las neuronas espejo están ligadas a la imitación de las estructuras sintácticas de los interlocutores con los que el ser humano interactúa a lo largo de su vida [37]. El área de Broca concretamente es la región que se activa durante la imitación y observación de la acción, así pues, quedaría refrendada la hipótesis evolutiva de que las neuronas espejo pueden ser elementos neuronales en la evolución del lenguaje. En esta línea cabe resaltar a la escritora Susan Blackmore, que en su libro "La máquina de los memes" sostiene que lo que nos distingue al ser humano de otros animales no es el lenguaje sino el imitar [38]. Andrew Meltzoff decía que la imitación era innata. La imitación recíproca (padresbebes) puede ser uno de los factores principales en la formación y fortalecimiento de las neuronas espejo del cerebro en desarrollo [39]. Carol Eckerman demostró la existencia de fuertes lazos entre la imitación y la comunicación verbal de los niños. Cuantos más juegos de imitación hace un niño cuando comienza a deambular más fluido será su lenguaje uno o dos años más tarde [40,41].

La relación existente entre la imitación y las neuronas espejo derivada de la intimidad que se establece entre el yo y el otro, puede considerarse como el primer paso hacia la empatía, que es uno de los pilares de la cognición social $[42,43]$.

Gallese fue pionero en proponer a las neuronas espejo como componente neuroanatómico de especial relevancia tanto para la comprensión como para la empatía respecto a las emociones de otras personas [44]. Lipps afirma que las neuronas espejo se activan tanto cuando la persona toma un objeto como cuando vemos que alguien toma un objeto, como si se estuviera dentro de la persona [45].

En la línea de lo expuesto, Niedenthal llegó a la conclusión que imitar a otros no es sólo una forma de comunicación no verbal, sino que también ayuda a percibir las expresiones de los otros y, por lo tanto de sus emociones [46]. Tanya Chartrand y John Bargh intentaron probar la hipótesis de que la capacidad de imitación de las personas y la empatía están muy relacionadas. Para poder probar esto, llevaron a cabo un experimento en la que hay un sujeto (que debe elegir determinadas fotografías) y un cómplice: en la primera parte del experimento el cómplice permanece al lado del sujeto y realiza determinados gestos; se observa que el sujeto termina realizándolos de forma inconsciente. En la segunda parte del experimento el cómplice imita los movimientos del sujeto o se mantiene neutro. Al finalizar esta segunda parte del experimento se le pregunta al sujeto si el participante que ha estado a su lado les había gustado. Se comprobó que cuando los sujetos habían sido imitados, las otras personas (los imitadores) les gustaban más. En la tercera parte del experimento se aplicada la primera parte del experimento solamente añadiendo 
un test de empatía al final de la prueba. Estos autores llegaron a la conclusión de que cuanto más realizaba el sujeto la acción que se parecía al cómplice más empatía sentía el sujeto por este. Lo que sugiere éste experimento es que a través de la imitación y la mímica, podemos sentir lo que otras personas están sintiendo, con lo cual podemos comprender sus estados emocionales [47].

Para poder reunir y fusionar la funcionalidad de las neuronas en espejo y la empatía debemos de considerar aquello que Marco Iacoboni propone acerca de la concepción de los mecanismos neuronales de la empatía: Las neuronas en espejo efectúan una imitación interna, o simulación, de la expresión facial observada. Envían señales al sistema límbico a través de la ínsula, y dicho sistema nos permite sentir la emoción que vemos [48].

\section{Componente cognitivo de la empatía}

Existen muchos estudios realizados con técnicas de neuroimagen, técnicas psicofisiológicas e incluso a lesionados medulares Tras la revisión de todos ellos podemos decir que todas las pruebas de neuroimagen nos llevan a fijarnos en los lóbulos frontales como la estructura más importante involucrada en el componente cognitivo de la empatía y la ToM. En concreto, podemos decir que son la corteza prefrontal media y la encrucijada temporo-parietal ETP [49, 50, 51, 52]. Fueron Fletcher y sus colaboradores los primeros en relacionar la ToM verbal y las áreas frontales mediales izquierda, en general en las áreas corticales 9/32 de Broadmann, que estaban ligadas a mentalización, aunque más tarde se sugirió que podían intervenir dichas estructuras en procesos de interacción social $[53,54,55,56]$. Aunque inicialmente se ha relacionado la función de la ToM como que es específica del lóbulo frontal derecho otros estudios posteriores de lesionados cerebrales han demostrado que también va ligado al izquierdo, por lo que la localización del ToM estaría ligada a la naturaleza verbal o no verbal del estímulo presentado [57, 58].

Podemos decir que hay otras estructuras que también juegan un papel importante en la habilidad para la comprensión de los estados mentales propios y de los otros, estas estructuras son: la corteza orbitofrontal y la corteza prefrontal ventromedial, el giro frontal medial izquierdo, la corteza cingulada posterior (CCP en adelante), el polo temporal y la amígdala. Se tienen algunos correlatos de cómo lesiones en la corteza orbitofrontal contribuye a la presentación de un déficit en la ToM. Los pacientes con lesiones bilaterales en dicha estructura, también pacientes que han sufrido una ablación del córtex orbitofrontal y sus conexiones con el sistema límbico sufren dicho déficit [59]. El resto de correlatos anatomofuncionales se han tomado de un artículo de revisión bibliográfica y se expone a continuación (véase tabla 1).

Tabla 2. Correlatos neurológicos de la ToM [59]

\begin{tabular}{|l|l|l|l|l|}
\hline Técnicas & Población & Proceso /Tarea & Localización cerebral & Autores \\
\hline RMNf & Sujetos con & Interpretación de & En los sujetos control se activan áreas de la & Baron- Cohen et \\
& autismo, Asperger & expresiones & corteza prefrontal, el giro temporal superior & al. (1999) \\
& y controles & emocionales a & y la amígdala. En cambio en los pacientes & con autismo o Asperger se activan regiones \\
& través de los ojos & & fronto-temporales y no así la amígdala. & \\
\hline
\end{tabular}




\begin{tabular}{|c|c|c|c|c|}
\hline RMN & $\begin{array}{l}16 \text { adultos (18-40 } \\
\text { años) y } 12 \text { niños } \\
\text { (8 a } 12 \text { años) }\end{array}$ & $\begin{array}{l}\text { Comprensión de } \\
\text { falsas creencias }\end{array}$ & Lóbulo frontal y ETP. & $\begin{array}{l}\text { Samson, } \\
\text { Aspperly, } \\
\text { Chiavarino y } \\
\text { Humpheys(2004) }\end{array}$ \\
\hline RMN & $\begin{array}{l}16 \text { adultos (18-40 } \\
\text { años) y } 12 \text { niños } \\
\text { (8 a } 12 \text { años) }\end{array}$ & $\begin{array}{l}\text { Tareas verbales } \\
\text { (historias) y no } \\
\text { verbales } \\
\text { (imágenes) de } \\
\text { falsa creencia }\end{array}$ & $\begin{array}{l}\text { En ambos grupos se observa actividad en la } \\
\text { Corteza prefrontal dorsolateral (en adelante } \\
\text { CPDL) derecha, giro frontal medial derecho, } \\
\text { la ETP bilateral y el giro occipital medial } \\
\text { derecho. En los niños se observa mayo } \\
\text { actividad en el giro temporal superior } \\
\text { derecho, el polo temporal derecho, cuneo y } \\
\text { la corteza prefrontal ventromedial (en } \\
\text { adelante CPVM) derecha. En los adultos se } \\
\text { observa mayor actividad en la amígdala } \\
\text { derecha }\end{array}$ & $\begin{array}{l}\text { Kobayashi et al } \\
(2007)\end{array}$ \\
\hline RMNf & $\begin{array}{l}12 \text { adultos de } 19 \text { a } \\
37 \text { años }\end{array}$ & $\begin{array}{l}\text { Faux Pas } \\
\text { (meteduras de } \\
\text { pata) }\end{array}$ & $\begin{array}{l}\text { Las historias que describen la violación de } \\
\text { normas sociales y situaciones embarazosas } \\
\text { activan regiones prefrontales mediales } \\
\text { orbitofrontales y regiones temporales }\end{array}$ & $\begin{array}{l}\text { Berthoz Armony } \\
\text { Blair y Dolan } \\
(2002)\end{array}$ \\
\hline PET & $\begin{array}{l}10 \text { sujetos con una } \\
\text { media de edad de } \\
24.7(6.18)\end{array}$ & $\begin{array}{l}\text { Inferencias sobre } \\
\text { estados mentales }\end{array}$ & $\begin{array}{l}\text { Las tareas que requieren inferir estados } \\
\text { mentales activan el lóbulo frontal media } \\
\text { izquierdo y el lóbulo temporal izquierdo }\end{array}$ & $\begin{array}{l}\text { Goel, Grafman, } \\
\text { Sadato, y Hallett } \\
\text { (1995) }\end{array}$ \\
\hline PET & $\begin{array}{l}8 \text { sujetos con una } \\
\text { media de edad de } \\
23.3(1.68)\end{array}$ & $\begin{array}{l}\text { Atribuciones de } \\
\text { intenciones a } \\
\text { otros }\end{array}$ & $\begin{array}{l}\text { Las tareas que requieren atribuir intenciones } \\
\text { a otros activan la CPM derecha, el córtex } \\
\text { prefrontal inferior derecho, el giro temporal } \\
\text { superior izquierdo, el cerebelo izquierdo, la } \\
\text { CCA bilateral y el giro temporal medio }\end{array}$ & $\begin{array}{l}\text { Brunet et al. } \\
(2000)\end{array}$ \\
\hline PET & $\begin{array}{l}6 \text { sujetos de } 24 \text { a } \\
65 \text { años }\end{array}$ & $\begin{array}{l}\text { Comprensión de } \\
\text { historias que }\end{array}$ & $\begin{array}{l}\text { Durante tareas de ToM y comprensión de } \\
\text { historias que no implican ToM, se activan } \\
\text { tres áreas en común: ambos polos } \\
\text { temporales, el giro temporal superior } \\
\text { izquierdo y la CCP. Sin embargo los test de } \\
\text { comprensión de historias específico activan } \\
\text { además un patrón específico que involucran } \\
\text { al giro frontal medial izquierdo (área } 8 \text { de } \\
\text { Brodmann) y la CCP. }\end{array}$ & $\begin{array}{l}\text { Fletcher et al. } \\
\text { (1995) }\end{array}$ \\
\hline
\end{tabular}




\begin{tabular}{|c|c|c|c|c|}
\hline PET & $\begin{array}{l}6 \text { sujetos de } 23 \text { a } \\
36 \text { años }\end{array}$ & $\begin{array}{l}\text { Comprensión de } \\
\text { historias verbales } \\
\text { y no verbales } \\
\text { (imágenes) que } \\
\text { implican ToM }\end{array}$ & $\begin{array}{l}\text { Ambas tareas activan la CPM y la ETP } \\
\text { bilateral. La tarea visual de asociación con } \\
\text { un incremento de actividad en regiones } \\
\text { adicionales como el precuneo, el giro frontal } \\
\text { medial y el cerebelo }\end{array}$ & $\begin{array}{l}\text { Gallagher et } \\
\text { al.(2000) }\end{array}$ \\
\hline PET & $\begin{array}{c}20 \text { adultos de } 18 \text { a } \\
31 \text { años }\end{array}$ & $\begin{array}{l}\text { Toma de } \\
\text { perspectiva: } \\
\text { emocional y } \\
\text { cognitiva }\end{array}$ & $\begin{array}{l}\text { Las diferentes condiciones de test de toma } \\
\text { de perspectiva (condición emocional y } \\
\text { condición cognitiva) involucran diferentes } \\
\text { regiones frontales: la condición emocional } \\
\text { activa preferencialmente la región medial } \\
\text { orbitofrontal, mientras que la condición } \\
\text { cognitiva activaría regiones más laterales y } \\
\text { anteriores del lóbulo frontal ventral. La } \\
\text { CPM y la EPT bilateral se activarían } \\
\text { durante ambas condiciones de toma de } \\
\text { perspectiva. }\end{array}$ & $\begin{array}{l}\text { Hynes Baird y } \\
\text { Grafton (2006) }\end{array}$ \\
\hline
\end{tabular}

Nota: RMNf= Resonancia Magnética Nuclear Funcional; RMN= Resonancia Magnética Nuclear; PET= Positron Emission Tomography.

\section{Conclusiones}

Como hemos visto a lo largo del estudio existen diferentes concepciones acerca de lo que es la empatía. Dependiendo de la época se ha pasado desde que a mediados del siglo XX se la considerase como una apreciación de los sentimientos de otros pero de forma totalmente automática y sin participación alguna hasta que, a finales del siglo XX se concibiera como una respuesta a los sentimientos de otras personas, y participarse en la interacción social. Hasta ya en la actualidad, en el siglo XXI considerarse como la percepción de los sentimientos y emociones de otras personas para la emisión de una respuesta de forma adecuada. Es en este último siglo cuando surgen los modelos multidimensionales, y en cuestión el que se ha estado abordando a lo largo de todo el artículo, en el que distinguen dos componentes de la empatía, el afectivo y el cognitivo.

En la actualidad lo que impera es la una visión multidimensional de la empatía en la que aparecen combinados dos componentes uno cognitivo y otro emocional, que están claramente diferenciados. Por un lado el componente emocional nos permite percibir y reconocer las emociones y por el otro, el componente cognitivo nos permite abstraer los procesos mentales de otras personas. Serían como dos componentes complementarios que actuarían de forma encadenada. Por un lado el componente emocional sería como el "órgano receptor" que se encarga de recibir la información del entorno y clasificarla / diferenciarla, es decir, percibir una información emocional a través de los gestos y del lenguaje del individuo y clasificarla en un tipo de emoción es (ira, tristeza, miedo, alegría, amor, etc.). A partir de ahí, y de forma conjugada 
con el componente emocional, entraría en juego el componente cognitivo que sería la parte racional que daría la interpretación de porque las otras personas se sienten así, integrando la información recibida de la parte emocional externa (empatía emocional) y la parte emocional interna del propio individuo procedente de la experiencia de circunstancias vividas previamente que guarden relación. Estas habilidades son de especial relevancia en nuestra vida social, puesto que permite al individuo compartir emociones, experiencias, necesidades y metas. Siendo por ello una herramienta básica y fundamental como habilidad social para la interacción, integración y adaptación del individuo en la sociedad.

En el presente estudio se muestran las estructuras cerebrales asociadas a la empatía emocional y cognitiva. Concluimos que en la literatura científica se han estudiado ambos componentes sin analizar la interacción entre ellos. Aunque nuestro estudio de revisión muestra ciertas limitaciones al no ser un estudio de revisión exhaustivo o meta-analítico, consideramos que futuros estudios deberían analizar la relación entre ambos componentes, así como estudiar el déficit en empatía asociados a diversas psicopatologías (autismo, demencias, depresión, esquizofrenia, daño cerebral, etc.) y los mecanismos cerebrales implicados.

\section{Bibliografía}

1. Diccionario. Real Academia Española. Vigésima, vol.1. 2001.

2. Lipps, T. Einfuhlung, innere Nachahmung und Organempfindung. Arch f d Psych 1903; 1, 465519.

3. Guilera Lládos T. Empatía. Conceptualización y bases neurobiológicas. Anales de psiquiatría. 2008; 24: 216-222.

4. Aring CD. Sympathy and empathy 1958; 167(4): 448-452.doi: 10.1001 / jama. 1958.02990210034008.

5. Rogers CR. A theory of therapy: Personality and inter- personal relationships as developed in the client-centred framework. En: Koch S, editor. Psychology, a study of science: Foundations of the person and the social con- Psychiatry 1989; 146: 1.

6. Hoffman ML. Is altruism part of human nature?. J Pers Soc Psychol. 1981 Jan; 40(1):121-37.

7. Zahn-Waxler C, Radke-Yarrow M. The origins of empathic concern. Motiv Emotion 1990; 14(2):107-30.

8. Gold JM, Rogers JD. Intimacy and isolation: A validation Study of Erikson's theory. Journal of Humanistic Psychology 1995; 35(1):78-86.

9. Mehrabian A. Manual for the Balanced Emotional Empathy Scale (BEES). Monterey, CA; 1996.

10. Cohen D, Strayer J. Empathy in conduct- disordered and comparison Routh. Dev Psychol 1996; 32: 988-998. 
11. Davis MH. Empathy: A social Psychological Approach. Social Psychology Series. Boulder: Westview Press; 1996.

12. Bohart AC, Greenberg LS. Empathy and psychotherapy: An introductory overview. In: LS Greenberg, editor. Empathy reconsidered: New directions for Psychotherapy. Washington, DC: American Psychological Association; 1997. p. 3-31.

13. Preston SD, De Waal FBM. Empathy: Its ultimate and proximate bases. BBS. 2002; 25(1): 1-20.

14. Gallese, V. The 'Shared Manifold' Hypothesis. From Mirror Neurons To Empathy. J Conscious Stud 2001; 8 (5-7), 33-50.

15. Hojat M, Gonnella JS, Nasca TJ, Mangione S, Vergare M, Magee M. Physician empathy: Definition, components, measurement, and relationship to gender and specialty. Am J Psychiatry 2002;159 (9):1563-9.

16. Hojat M, Gonnella JS, Mangione S, Nasca TJ. Empathy in medical students as related to academic performance clinical competence and gender. Med Educ 2002; 36: 522-7.

17. Baron- Cohen S, Richler J, bisaya D, Gurunathan N, Wheelwright S. The systemizing quotient: An investigation of adult with Asperger syndrome or high-functioning autismo, and normal sex diferences. Phil Trans R Soc Lond B 2003; 358(1430):361-74.

18. González de Rivera JL. Empatía y ectapía. Psiquis 2004; 25(6):243-5

19. Decety J, Jackson P L. The functional architecture of human empathy. Behav Cogn Neurosci Rev 2004; 3: 71-100.

20. Blair R J R, Budhani S, Colledge E, Scott SK. Deafness to fear in boys with psychopathic tendencies. J Child Psychol Psychiatry 2005; 46: 327-336.

21. Decety J, Lamm C. Human empathy through the lens of social neuroscience. Scientific World Journal 2006; 6: 1146-1163

22. Preston SD, Bechara A, Damasio H, Grawski TJ, Stansfield RB, Metha S, et al. The neural substrates of cognitibe empathy. Soc Neurosci 2007; 2: 254-75.

23. Shamay- Tsooy SG, Aharon-Peretz J, Perry D. Two Systems for empathy: a double dissociation between emocional and cognitive empathy in inferiro frontal gyrus versus ventromedial prefrontal lesions. Brain 2009; 132: 617-27.

24. Spinella M. Prefrontal substrates of empathy: psychometric evidence in a community sample. Biol Psychol 2005; 70:175-81.

25. Blair RJ. Dissociable system for empathy. Nivartis Found Symp 2007; 278:134-41. 
26. Moya- Albiol L, Herrero N, Bernal MC. Bases neuronales de la empatía. Rev Neurol 2010; 50 (2): 89-100.

27. Geday J, Gjedde AS, Kupers R. Emotional valence modulates activity in the posterior fusiform gyrus and the inferior medial prefrontal cortex in social perception. Neuroimage 2003; 18: 675-84.

28. Carr L, Iacoboni M, Dubeau MC, Mazziotta JC, Lenzi GL. Neural mechanisms of empathy in humans: a relay from neural Systems for imitation to límbico areas. Proc Natl Acad Sci USA 2003; 100: 597-502.

29. Keysers C, Wicker B, Gazzola V, Anton JL, Fogassi L, Gallese V. A touching sight: SII/PV activation Turing the observation and experience of Couch. Neuron 2004; 42: 335-46.

30. Philips Ml, Young AW, Senior C, Brammer M, Andrew C, Calder AJ, et al. A specific neural substrate for perceiving facial expressions of disgust. Nature 1997; 389: 495-8.

31. Keysers C, Gazzola V. Towards a unifying neural theory of social cognition. Prog Brain Res 2006; 156: 379-401.

32. O`Doherty J, Deichmann R, Critchley HD, Dolan RJ. Neural responses Turing anticipation of primary taste reward. Neuron 2002; 33:815-26.

33. Sanchez-Navarro JP, Román F. Amígdala, corteza prefrontal y especialización hemisférica en la experiencia y expresión emocional. An de psicología 2004; 20 (2): 223-240.

34. Alibali M W, Helth DC, Myers HJ. "Effects of visibility between speaker and listener on gestare production: Some gestures are meant to be seen". J Mem Lang 2001; 44:169-188.

35. Molnar- Szakacs IS, Wilson M, Iacoboni M. "I see what you are saying: The neuronal correlatos of gestare perception”. Número de programa 1287.2055 Abstract Viewer, CD-ROM. Washinton, DC, encuentro de la Society for Neuroscience.

36. Rizolatti G, Arbib MA.“Language within our grasps”. Nat Rev Neurosci 1998; 21:188-194.

37. Hartfield E, Cacioppo JT, Rapson RL. Emotional cognition. Nueva York: Cambridge University Press; 1994.

38. Blackmore S. La máquina de los memes. Barcelona: Paidos Iberica; 2000.

39. Meltzoff A N, Moore MK. "Imitation of facial and manual gestures by human neonatos". Science 1977; 198: 74-78.

40. Meltzoff A N, Prinz W. The imitative mind: Development, evolution, and brain bases. New York: Cambridge: University Press; 2002. 
41. Eckerman CO, Didow SM. "Nonverbal imitation and toddlers'mastery of verbal means of achieving coordinated actions". Dev Psychol 1996; 32:141-152.

42. Roger S J, Pennington B. "A theoretical approach to the deficits in infantile autism. Dev Psychol $1991 ; 3: 137-162$.

43. Whiten A, Brown JD."Imitation and the Redding of other minds: Perspectives from the study of autism, normal children and non-human primates" In Braten S. ed. Intersubjetive Communications and emotion in early ontogeny. Cambridge: Cambridge University Press; 1999. p. 260-280.

44. Gallese V. “The shared manifold hypothesus”. J Conscious Stud 2001; 8: 33- 50.

45. Lipps T. "Einfülung, innere nachahmung und orgabebenpfindung”. Arch F Gs- Psy1903;1(2).

46. Niedenthal PM, Barsalou LW, Winkielman P et al. "Embodiment in attitudes social perception and emotion”. Pers Soc Psychol Rev 2005; 8: 184-211.

47. Chartrand TL, Bargh JA. "The chameleon effect: The perception-behavior link and social interaction. Pers Soc Psychol Rev 1999; 76: 893-910.

48. Iacoboni M. Las neuronas espejo: Empatía, neuropolitica, autismo, imitación, o de cómo entendemos a los otros. España: Katz; 2009.

49. Tirapu Ustarróz J, Ríos Lago M, Maestú Unturbe F .Manual de Neuropsicología. Barcelona: Vigueras; 2012.

50. Brunet E, Sarfati Y, Hardy-Bayle MC, Decety J. A PET investigation of the attribution of intentions with a nonverbal task. Neuroimage 2000; 11: 157-166.

51. Frith U, Frith C D. Development and neurophysiology of mentalizing. Philos Trans R Soc Lond B Biol Sci 2003; 358: 459-473.

52. Saxe R, Schulz LE, Jiang Y V. Reading minds versus following rules: Dissociating theory of mind and executive control in the brain. Soci Neurosci 2006; 1:284-298.

53. Fletcher PC, Happé F, Frith U, et al. Other minds in the brain: a functional Imaging study of "theory of mind" in story comprensión. Cognition 1995; 57:109-28.

54. Den Ouden HE, Frith U, Frith C, et al. Thinking about intentions. Neuroimage 2005; 28:787-96.

55. Vollm BA, Tayloe AN, Richarson P, et al. Neuronal correlatos of theory of mind and empathy: a funcional magnetic resonante Imaging study in a noverbal task. Neuroimage 2006; 29:90-8.

56. Gallagher HL, Frith CD. Funtional Imaging of "theory of mind". Trends Cogn Sci 2003; 7:77-83. 
57. Stuss DT, Gallup JR, Alexander MP. The fronal lobes are necessary for "theory of mind". Brain 2001; 124: 279-86.

58. Brunet E, Sarfati Y, Hardy-Bayle MC y Decety J. A PET investigation of the attribution of intentions with a nonverbal task. Neuroimagen 2000; 11: 157-166.

59. Siegal M, Varley R. Neural Systems envolved in "theory of ind". Nat Rev Neurosci 2002; 3: 46371.

60. Stone VE, Baron-Cohen S, Calder A, et al. Acquired theory of mind impairments in individuals with bilateral amygdala lesions. Neuropsychologia 2003; 41: 209-20.

61. Adolphs R. How do we Know the minds of others? Domain-specificity, simulation, and inactive social cognition. Brain Res 2006; 1079: 25-35.

62. Filippetti VA, López MB, Richaud MC. Aproximación neuropsicológica al constructo de Empatía: Aspectos Cognitivos y Neuroanatómicos. Cuadernos de Neuropsicología/Panamerican Journal of Neuropsychology 2012; 6: 63-83. 